\title{
Losing Control: Sleep Deprivation Impairs the Suppression of Unwanted Thoughts
}

II

\author{
Marcus O. Harrington' ${ }^{1}$, Jennifer E. Ashton ${ }^{1}$, \\ Subbulakshmi Sankarasubramanian ${ }^{2}$, Michael C. Anderson ${ }^{2}$, \\ and Scott A. Cairney ${ }^{1,3}$ \\ ${ }^{1}$ Department of Psychology, University of York; ${ }^{2}$ Medical Research Council Cognition and Brain Sciences \\ Unit, University of Cambridge; and ${ }^{3}$ York Biomedical Research Institute, University of York
}

\begin{abstract}
Unwanted memories often enter conscious awareness when individuals confront reminders. People vary widely in their talents at suppressing such memory intrusions; however, the factors that govern suppression ability are poorly understood. We tested the hypothesis that successful memory control requires sleep. Following overnight sleep or total sleep deprivation, participants attempted to suppress intrusions of emotionally negative and neutral scenes when confronted with reminders. The sleep-deprived group experienced significantly more intrusions (unsuccessful suppressions) than the sleep group. Deficient control over intrusive thoughts had consequences: Whereas in rested participants suppression reduced behavioral and psychophysiological indices of negative affect for aversive memories, it had no such salutary effect for sleep-deprived participants. Our findings raise the possibility that sleep deprivation disrupts prefrontal control over medial temporal lobe structures that support memory and emotion. These data point to an important role of sleep disturbance in maintaining and exacerbating psychiatric conditions characterized by persistent, unwanted thoughts.
\end{abstract}

\section{Keywords}

sleep deprivation, emotion regulation, perseverative thoughts, memory control, psychiatric disorders, open data

Received 7/17/19; Revision accepted 5/11/20

Memories of unpleasant experiences and thoughts can intrude into conscious awareness when reminders to them are confronted. Individuals suffering from psychiatric conditions such as posttraumatic stress disorder (PTSD) and major depressive disorder (MDD) typically experience a disproportionate number of unwanted memory intrusions, and difficulties in limiting the duration and recurrence of these intrusions compound negative mood and affective dysregulation (Brewin, Gregory, Lipton, \& Burgess, 2010; Mihailova \& Jobson, 2018; Moritz et al., 2014; Newby \& Moulds, 2011; Payne, Kralj, Young, \& Meiser-Stedman, 2019). One's capacity for inhibiting intrusive thoughts might therefore play a fundamental role in maintaining mental health and well-being (Gagnepain, Hulbert, \& Anderson, 2017).

The ability to control intrusive memories and thoughts can be studied in the laboratory by measuring people's success at suppressing memory retrieval when confronted with reminders to unwanted thoughts. For example, one widely used task, known as the think/ no-think (TNT) paradigm (Anderson \& Green, 2001), requires participants to either actively engage (think) or suppress (no-think) memory retrieval when presented with reminder cues to associated memories, often aversive images. An intrusion occurs when participants' attempts to suppress retrieval during no-think trials fail and the reminder cue triggers an involuntary retrieval of the associated memory. Reminder cues typically elicit an intrusion less than $50 \%$ of the time (Gagnepain et al., 2017; Hellerstedt, Johansson, \& Anderson, 2016; Levy \& Anderson, 2012; van Schie \& Anderson, 2017), but people vary widely in memory

\section{Corresponding Author:}

Scott A. Cairney, Department of Psychology, University of York

E-mail: scott.cairney@york.ac.uk 
control ability (Levy \& Anderson, 2008). The factors contributing to this variability are poorly understood. Identifying determinants of successful retrieval suppression could contribute to the understanding of vulnerability to disorders characterized by intrusive thoughts (Brewin et al., 2010; Mary et al., 2020; Moritz et al., 2014; Streb, Mecklinger, Anderson, Johanna, \& Michael, 2016).

Retrieval suppression ability is thought to be intrinsically linked to inhibitory control. According to the inhibitory deficit hypothesis (Levy \& Anderson, 2008), individual differences in regulating intrusive memories originate from variation in underlying inhibition function. This hypothesis predicts that conditions that strain inhibitory control will likewise undermine the ability to suppress unwanted thoughts. In healthy adults, sleep deprivation impairs cognitive functioning (Alhola \& Polo-Kantola, 2007; Walker, 2009; Wild, Nichols, Battista, Stojanoski, \& Owen, 2018), especially executive control (Drummond, Paulus, \& Tapert, 2006; Nilsson et al., 2005), making sleep loss an important candidate factor mediating fluctuations in thought control. Indeed, mental fatigue arising from sustaining an effortful task can increase the frequency of intrusions during the TNT task (van Schie \& Anderson, 2017). Moreover, the prefrontal-medial temporal lobe (MTL) networks involved in retrieval suppression (Benoit, Hulbert, Huddleston, \& Anderson, 2015; Gagnepain et al., 2017; Levy \& Anderson, 2012) are disrupted by sleep loss (Yoo, Gujar, Hu, Jolesz, \& Walker, 2007), which suggests that losing sleep may heighten people's vulnerability to intrusive thoughts (Chee, 2004; Mazur, Pace-Schott, \& Hobson, 2002; Thomas et al., 2000, 2003; van Schie \& Anderson, 2017; Yoo et al., 2007). Chronic sleep disturbance is also a formal symptom of most psychiatric conditions, particularly PTSD (Maher, Rego, \& Asnis, 2006) and MDD (Riemann, Berger, \& Voderholzer, 2001).

These observations led us to consider whether the established link between psychiatric conditions and disturbed sleep may be mediated partially by sleep deficits compromising a person's ability to regulate emotion by suppressing retrieval of aversive thoughts. For example, suppressing retrieval of aversive scenes reduces people's emotional response to those scenes later on, as revealed by changes in subjective affect ratings for the suppressed stimuli and the relationship of those changes to prefrontally driven down-regulation of the amygdala during memory intrusions (Gagnepain et al., 2017). This impact of retrieval suppression on perceived emotion (referred to hereafter as affect suppression) suggests that retrieval suppression contributes to affective homeostasis by reducing the negative tone of unpleasant events. If sleep loss compromises memory control, it may diminish the impact that retrieval suppression has on affective responses to unwanted thoughts, a possibility consistent with the well-documented negative consequences of sleep loss on mood (Dinges et al., 1997; Short \& Louca, 2015; Zohar, Tzischinsky, Epstein, \& Lavie, 2005). Whether sleep loss disrupts affect suppression arising from memory control and whether affect suppression effects are mirrored in psychophysiological reactivity to suppressed thoughts have never been examined.

To determine whether sleep deficits could contribute to the pathogenesis and maintenance of intrusive symptoms, we investigated the impact of sleep deprivation on memory control in healthy adults. Participants learned associations between faces and emotionally negative or neutral scenes before an overnight interval of sleep or total sleep deprivation (Fig. 1a). The following morning, participants completed a TNT task for the face-scene associations (Fig. 1b). On each trial of this task, a face was presented alone in either a green or red frame, instructing participants to either actively retrieve (think) or suppress (no-think) the associated scene, respectively. Attempts to suppress retrieval of the scene often initially fail, leading the scene to intrude into participants' awareness involuntarily despite efforts to stop it. Because involuntary retrievals in the TNT paradigm are, as in real life, unobservable events private to the individual, it was necessary to identify their occurrence through intrusion reports. After each trial, participants reported, using a 3-point scale (never, briefly, often), whether the associated scene had entered awareness. We then quantified variations in memory control success by using the proportion of no-think trials that triggered any awareness of the associated scene, reflecting a momentary failure of retrieval suppression (i.e., reports of briefly or often; referred to hereafter as intrusions).

Intrusion reports provide a validated index of involuntary retrieval. Researchers using functional MRI (fMRI) to measure intrusion reports have established that in rested individuals, intrusion trials (unsuccessful suppression attempts) trigger greater activation in the right dorsolateral prefrontal cortex (rDLPFC), greater negative coupling of rDLPFC with both the hippocampus and amygdala, and greater down-regulation of activity in the latter structures, consistent with a reactive engagement of top-down inhibitory control to suppress the intrusive content (Benoit et al., 2015; Gagnepain et al., 2017; Levy \& Anderson, 2012). Moreover, research in which electroencephalogram (EEG) was used established that reports of intrusions are associated with a brief increase in event-related-potential markers of working memory storage (Hellerstedt et al., 2016), followed by their rapid elimination, consistent with a reactive purging of the intrusion. In contrast, trials that attracted intrusion reports of never (successful suppression 


\section{a}

\section{Study Procedure}
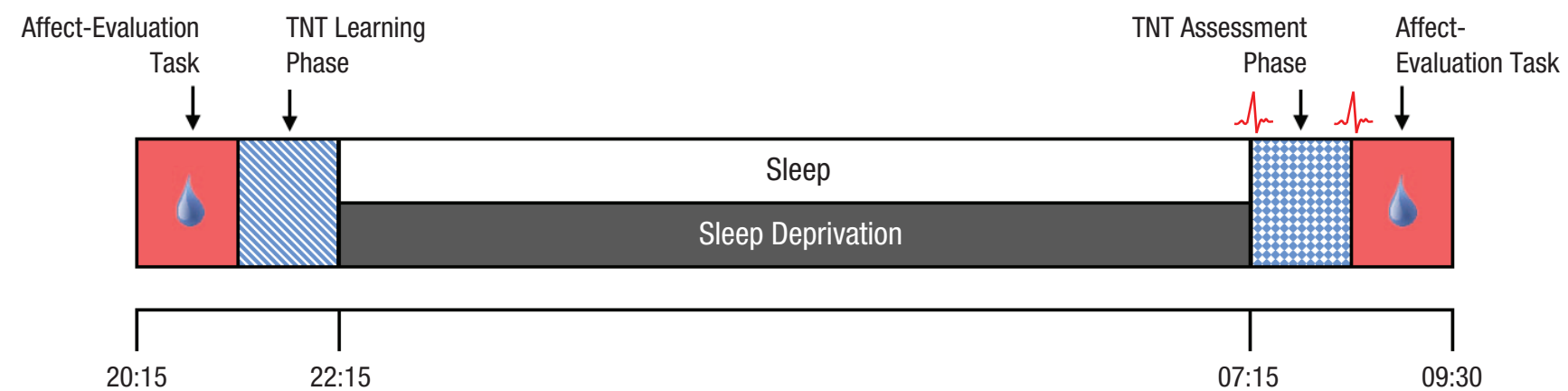

b

\section{TNT Task}

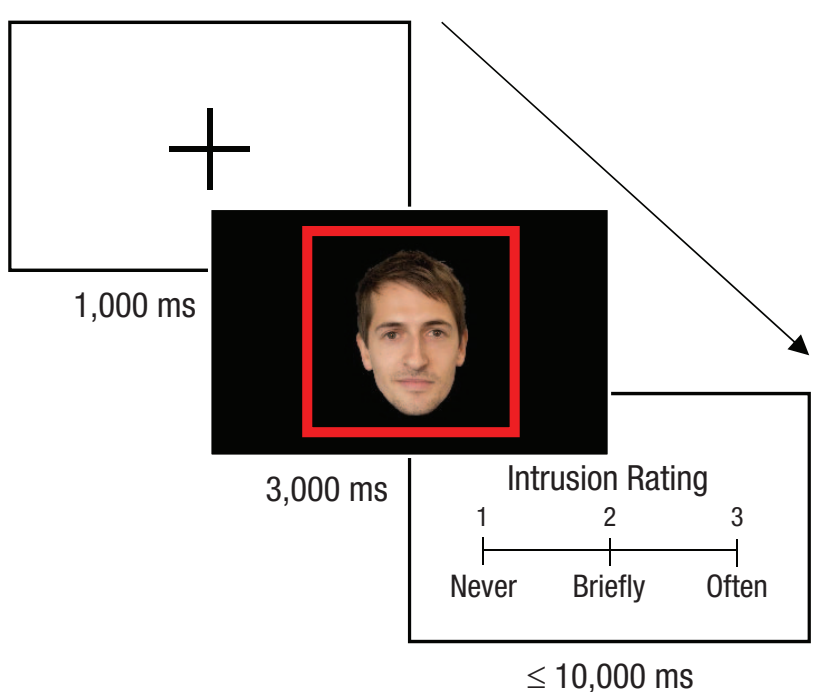

C

\section{Affect-Evaluation Task}

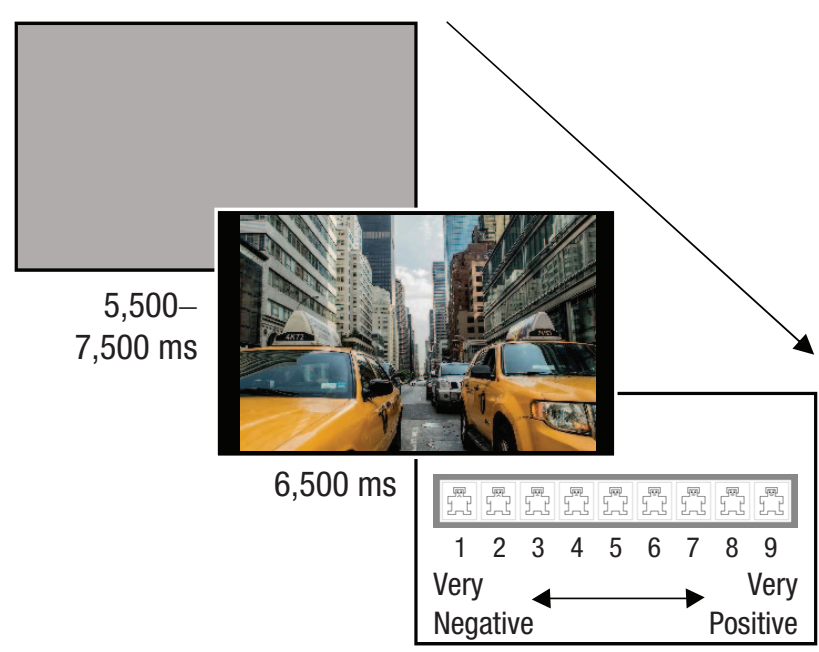

$\leq 15,000 \mathrm{~ms}$

Fig. 1. Study procedures and tasks. (a) All participants completed Session 1 in the evening, which included the first of two affect-evaluation tasks and the learning phase of the think/no-think (TNT) task. Participants in the sleep group slept in the sleep laboratory for $\sim 8$ hr. Participants in the sleep-deprivation group remained awake across the night. All participants then completed Session 2, which included the assessment phase of the TNT task and the second affect-evaluation task. We collected skin-conductance responses (SCRs) to scene presentations throughout the affect-evaluation tasks. We recorded resting heart rate variability (HRV) before and after the TNT assessment phase. (b) In the critical TNT assessment phase, we presented participants with faces in red or green frames. For red-framed faces (no-think trials), we instructed participants to avoid thinking about the associated scene without generating diversionary thoughts; for green-framed faces (think trials), we instructed them to visualize the associated scene. After each trial, participants reported the extent to which they thought about the paired scene (never, briefly, or often). We considered reports of briefly or often to be intrusions. (c) In the affect-evaluation task, participants viewed negative and neutral scenes, and we asked them to provide an emotional rating for each image on a scale from 1 (very negative) to 9 (very positive).

attempts) instead have shown significant increases in beta frequency power, consistent with successful proactive control of retrieval (Castiglione, Wagner, Anderson, \& Aron, 2019). Given these intrusion indices, we predicted that sleep-deprived participants would report more intrusions for no-think scenes than would participants who slept and would exhibit impaired ability to reduce the frequency of intrusions over time.
Studies that employ the TNT paradigm often measure the consequences of thought suppression for memory accessibility via a recall test administered after the TNT assessment phase. These studies have shown that suppressing a memory often impairs its later accessibility (suppression-induced forgetting; for review, see Anderson \& Hanslmayr, 2014). However, our primary objective here was instead to test how sleep deprivation influenced the 
ability to down-regulate unwanted thoughts and, consequently, affect suppression for the intruding content. To investigate how the predicted failures in intrusion control following sleep loss influenced affect suppression, we acquired emotional ratings for the scenes both before the overnight delay and also after the TNT phase the following morning (Fig. 1c). We also measured SCRs to scene presentations to examine whether psychophysiological measures of sympathetic arousal mirrored suppression-induced changes in subjective affect. We predicted that retrieval suppression would attenuate both subjective and psychophysiological reactivity to negative scenes in the sleep group. Critically, however, if our hypothesis about the role of sleep loss in the pathogenesis of psychiatric disorders is correct, this salutary effect of suppression on negative affect should be significantly reduced in otherwise healthy participants randomly assigned to our sleep-deprivation group.

We also measured the physiological correlates of memory control by collecting heart rate variability (HRV) recordings before and after the TNT assessment phase. Spectral analysis of HRV has previously identified two reliable components: high-frequency HRV (HF-HRV; 0.15-0.40 Hz) and low-frequency HRV (LF-HRV; 0.04$0.15 \mathrm{~Hz}$ ). Note that higher HF-HRV is linked to superior executive functioning (for review, see Thayer \& Lane, 2009), including memory control (Gillie, Vasey, \& Thayer, 2014), whereas LF-HRV instead increases with fatigue (Tran, Wijesuriya, Tarvainen, Karjalainen, \& Craig, 2009). Given these observations and given the hypothesized disruption to inhibitory control with sleep deprivation, we predicted that higher HF-HRV would be associated with superior affect suppression and intrusion control in the sleep group but not the sleep-deprivation group. We further predicted significantly higher LF-HRV following sleep deprivation than after a night of sleep, providing physiological confirmation of extreme fatigue.

\section{Method}

\section{Participants}

Sixty healthy individuals were recruited for this study via an online recruitment system and randomly assigned to a sleep group $(n=30)$ or a sleep-deprivation group $(n=30)$. Of these participants, one was excluded for failing to follow task instructions. The reported data relate to the remaining 59 participants (sleep group: $n=29,13$ men, mean age $=19.79$ years, $S D=1.63$ years; sleep deprivation group: $n=30,12$ men, mean age $=20.20$ years, $S D=1.75$ years) who participated in return for $£ 40$ payment or bachelor's-level psychologycourse credit (University of York). To reduce demand characteristics, we advertised and framed the study to participants as an investigation into the effect of sleep and sleep deprivation on attention.

Participants reported no history of neurological, psychiatric, attention, or sleep disorders, and they typically rose by 8:00 a.m. after at least $6 \mathrm{hr}$ of sleep, as indicated by self-report. Beck Depression Inventory (BDI-II; Beck, Steer, \& Brown, 1996) scores (collected at the end of the experiment) did not differ significantly between groups (sleep group: $M=7.52, S E M=1.08$; sleep-deprivation group: $M=5.30, S E M=0.97), t(57)=1.53, p=.13$. We requested that participants refrain from consuming alcohol or caffeine for $24 \mathrm{hr}$ before the experiment. Wristwatch actigraphy was used to ensure that participants did not nap during the day preceding the overnight phase. Written informed consent was obtained from all participants in line with the requirements of the University of York's Department of Psychology Research Ethics Committee, which approved the study.

\section{Stimuli}

Forty-eight emotionally neutral face images (half men, half women) served as cues in the TNT task. An equal number of scene images (half negative, half neutral) selected from the International Affective Picture System (IAPS; Lang, Bradley, \& Cuthbert, 2005) served as targets. Negative scenes received lower affect ratings compared with neutral scenes from participants in the current study during the first affect-evaluation task, $F(1$, 57) $=630.89, p<.001, \eta_{p}{ }^{2}=.92$, demonstrating that the scenes elicited the expected emotional response. Facescene pairs were created by randomly assigning each face cue to a target scene. Three lists of 16 pairs (eight negative, eight neutral) were created from the 48 pairs to allow three within-subjects TNT conditions (think, no-think, baseline). The assignment of pairs to TNT conditions was counterbalanced across participants for each of the sleep groups. Twelve additional face-scene pairs (six negative, six neutral) were created to serve as fillers that were also used in the practice phases. The same 60 scenes (48 experimental +12 practice) were used for the affect-evaluation tasks, which featured an additional eight filler scenes (four negative, four neutral).

\section{Procedure}

A schematic representation of the study procedure is shown in Figure 1a. Participants completed two sessions that were separated by an overnight delay containing either sleep or sleep deprivation. At 9:00 a.m. on the first day, participants collected an actigraphy watch that they wore until 8:15 p.m. when they arrived at the University of York's Emotion Processing and Offline Consolidation laboratory. 
Affect-evaluation task. Participants provided emotion ratings for 68 IAPS scene images using a pictorial scale that ranged from a frowning face on the far-left side of the scale to a smiling face on the far-right side of the scale (Fig. 1c). On each trial, a scene was presented for 6.5 s. Participants were asked to focus their attention on the scene for the entire time it was on the screen. Following a 2-s blank-screen delay, the affect rating scale was displayed prompting participants to provide their rating on a scale from 1 (corresponding to the frowning face on the far left side of the scale) to 9 (corresponding to the smiling face on the far right side of the scale). Participants were told that the extreme left side of the scale should be used for scenes that made them feel completely unhappy, annoyed, unsatisfied, melancholic, despaired, or bored and that the extreme right side of the scale should be used for scenes that made them feel completely happy, pleased, satisfied, contented, and hopeful. Participants were given $15 \mathrm{~s}$ to provide their affect rating for each picture, although they were asked to respond quickly and spontaneously. The trial terminated once an affect rating had been provided or the 15-s time limit expired, and participants then viewed a blank screen for $5 \mathrm{~s}$, followed by a fixation cross for a random interval of either $0.5 \mathrm{~s}, 1$ $\mathrm{s}, 1.5 \mathrm{~s}$, or $2 \mathrm{~s}$, followed by the next scene. This pattern continued until all scenes had been viewed and rated. The affect-evaluation task was repeated after the TNT assessment phase. SCRs were recorded throughout the affect-evaluation tasks.

TNT task. Next, participants completed the learning phase of the TNT task. Participants initially encoded facescene pairs by studying them one at a time for $6 \mathrm{~s}$. To reinforce learning and ensure adequate knowledge of the face-scene pairs, we then had participants complete a test phase in which faces were displayed individually for up to $4 \mathrm{~s}$ and participants indicated whether they were able to fully visualize the associated scene. If participants indicated that they were able to visualize the associated scene, they were next presented with the correct scene alongside two additional foil scenes that they had seen previously in the learning phase but were not paired with that particular face. Participants were required to select the scene associated with the face. If participants failed to identify the correct scene, or they indicated that they could not visualize the scene associated with the face, their memory for the face-scene pair was probed again later in the test phase. Regardless of whether participants were able to correctly identify the scene paired with a particular face, the correct face-scene pairing was presented for $3.5 \mathrm{~s}$ after each trial. Participants were instructed to use this feedback as an opportunity to reinforce their knowledge of the pairs. The test phase continued until each face-scene pair had been correctly identified once.
Following completion of the test phase, a second, identi$\mathrm{cal}$, test phase was administered to reinforce learning. This overtraining procedure was employed to ensure that participants would experience difficulty in preventing scenes from intruding into consciousness when presented with face cues on no-think trials of the TNT assessment phase.

Before the overnight delay, participants carried out a mock TNT assessment phase. Here, we provided detailed and comprehensive training on the TNT task, allowing participants to practice inhibiting memory intrusions and calibrate their intrusion reports for the critical TNT assessment phase the following morning. In the mock task, participants completed 24 trials (12 think, 12 nothink), which used 12 filler items (each suppressed or retrieved twice) that were different to those used in the TNT assessment phase proper. Participants then went to sleep or remained awake overnight; details about the overnight delay are provided below.

The following morning, participants completed a memory refresher phase in which the face-scene pairs were presented for $1.5 \mathrm{~s}$ per pair, offering participants the opportunity to reinforce their knowledge of the pairs. The TNT assessment phase proper was then administered in five blocks, each lasting approximately 8 min. During each block, two repetitions of 16 think (eight negative and eight neutral face cues) and 16 nothink (eight negative and eight neutral face cues) items were presented in pseudorandom order, with the two repetitions of each item appearing at least three trials away from each other. Accordingly, participants completed a total of 320 trials ( 32 trials $\times 2$ conditions $\times 5$ blocks). Face cues appeared inside a green frame on think trials and inside a red frame on no-think trials. For green-framed faces, participants were instructed to visualize, in as much detail as possible, the scene associated with the face for the entire $3 \mathrm{~s}$ that it was on the screen. For red-framed faces, participants were instructed to focus their attention on the face for the entire $3 \mathrm{~s}$ but simultaneously prevent the associated scene from coming to mind (Fig. 1b). In keeping with earlier work (e.g., Gagnepain et al., 2017; Mary et al., 2020), participants were told to accomplish this by making their mind go blank rather than by replacing the unwanted scene with another image, thought, or idea. If the scene came to mind automatically, participants were asked to actively push the scene out of mind.

Immediately after each face cue presentation, participants reported whether the associated scene had entered awareness (during the time that the face was on screen) by pressing a key corresponding to one of three options: never, briefly, and often. Participants were instructed to provide a response of briefly if the scene had briefly entered conscious awareness at any 
time during the trial. They were told that responses of often should be provided if the scene had entered awareness several times during the trial or once but for a longer period of time than would be considered brief. Participants were given up to $10 \mathrm{~s}$ to make this rating; however, they were instructed to provide their rating quickly without dwelling on their decision. Participants moved immediately onto the next trial after providing their intrusion rating. These intrusion ratings were collected to ascertain how competent participants were at suppressing scenes associated with faces for no-think trials. Although we collected intrusion reports on a 3-point scale (never, briefly, often) for every trial, in practice, people rarely give ratings of often on no-think trials $(M=3.15 \%$ of no-think trials in the current study, $S E M=0.62 \%$ ). For simplicity, we therefore followed prior work by combining the briefly and often responses (Benoit et al., 2015; Gagnepain et al., 2017; Hellerstedt et al., 2016; Levy \& Anderson, 2012; van Schie \& Anderson, 2017), rendering the judgment binary (intrusion or nonintrusion). Participant responses were followed by a jittered fixation cross lasting between 0.5 and $9 \mathrm{~s}$ before the next face cue was presented.

Note that 16 scenes that were included in the TNT learning phase and the affect-evaluation task did not appear in the TNT assessment phase. These baseline scenes provided an estimate of generalized changes in affect that could be compared with changes resulting from mnemonic suppression (no-think scenes).

Recognition task. In the morning, after the second affectevaluation task, memory for all face-picture pairs was examined using a recognition test. For procedure and results for this task, see Recognition Task S1 in the Supplemental Material available online.

Overnight delay. Electrodes were attached to participants in the sleep group following completion of the first session to allow for overnight polysomnographic recording. Lights were turned out at approximately 10:45 p.m., and participants were awoken at 6:45 a.m., providing an 8-hr sleep opportunity. Before the morning session began, participants were given the opportunity to shower and eat breakfast.

Participants in the sleep-deprivation condition remained awake in a university seminar room throughout the overnight delay under the supervision of at least one researcher. Participants were tested in groups of two or three; they were permitted to communicate, read, use the PC, watch TV, or play games. Snacks were provided, but participants were not permitted to consume caffeine. Once the second session began, participants had been awake for $>24 \mathrm{hr}$.

\section{Equipment and data processing}

Behavioral. All aspects of the TNT task were written and implemented using Presentation (Neurobehavioral Systems, Albany, CA). The affect-evaluation task was administered with E-Prime (Psychology Software Tools, Pittsburgh, PA). Behavioral tasks ran on a desktop computer, and visual aspects were displayed on a flat-screen monitor. Participant responses were collected using the computer keyboard. Behavioral data were analyzed using IBM SPSS 24.

Intrusion-proportion scores. Intrusion ratings provided during the TNT assessment phase were used to classify each trial as either eliciting an intrusion (briefly or often responses) or not (never responses) in a binary fashion (Benoit et al., 2015; Gagnepain et al., 2017; Hellerstedt et al., 2016; Levy \& Anderson, 2012; van Schie \& Anderson, 2017). Unwanted retrieval events were separated into categories of briefly and often to ensure that all intrusions, irrespective of their persistence or strength, were reported. We calculated the proportion of trials that evoked a memory intrusion separately for each participant, trial block (1-5), TNT condition (think, no-think), and scene valence (neutral, negative). Overall intrusion-proportion scores were also calculated by averaging mean intrusion-proportion scores across trial blocks separately for each TNT condition and scene valence.

Intrusion slope scores. To measure changes in intrusion frequency for no-think trials across TNT blocks, we calculated proportionalized intrusion slope scores separately for each participant and scene valence (neutral, negative). Slope scores were calculated by taking the slope of the intrusion frequencies across the five TNT trial blocks. This value was divided by intrusion frequency in the first block to account for the fact that initial intrusion rates varied and participants with more initial intrusions had more room to decrease their intrusion frequency. We then multiplied the values by -1 to render the (primarily) negative scores positive. Increasing positive scores reflect increasing levels of control at down-regulating the frequency of intrusions. This measure was $z$ normalized within the participant's face-scene pair counterbalancing group (Hellerstedt et al., 2016; Levy \& Anderson, 2012). The $z$ normalizing within each counterbalancing group allowed us to quantify a participant's intrusion slope score with respect to a group of participants receiving precisely the same stimuli in the TNT task.

Relapse probability. Each item that appeared in the TNT task was presented 10 times (twice in each TNT block). This repetition allowed us to investigate how well 
a participant's efforts to suppress a particular item on one trial carried forward to the next trial involving that same item. The frequency of relapses for no-think items was calculated as the number of no-think trials in repetitions 2 through 10 in which an item elicited an intrusion but that specific item had been successfully suppressed on its immediately preceding presentation. This was done separately for each participant, trial transition (1 to 2, 2 to $3, \ldots 9$ to 10 ), and scene valence (neutral, negative). Each value was divided by the number of successfully suppressed items (nonintrusions) during the first repetition of that trial transition (e.g., repetition 3 of transition 3 to 4 ) to produce relapse probability scores (van Schie \& Anderson, 2017). This was to account for the fact that individuals with fewer intrusions on the first repetition of each trial transition had greater relapse potential.

Affect suppression. Affect ratings gathered during the affect-evaluation tasks were used to measure overnight changes in subjective emotional reactivity to the scenes. Mean affect rating values were calculated for each participant, session (predelay, postdelay), TNT condition (think, no-think, baseline), and scene valence (neutral, negative). Affect suppression scores were then calculated by subtracting the averaged values at Session 1 from those at Session 2. To account for individual differences in emotional responses at Session 1, we divided affect suppression scores in each condition by the mean affect rating at Session 1 to produce proportionalized affect suppression scores. Greater scores reflect more positive affect evaluations at Session 2 compared with Session 1.

Electrodermal activity. Electrodermal activity recorded during the affect-evaluation tasks was used to measure overnight changes in SCRs to the presentation of scenes. In Sessions 1 and 2, the mean SCR was calculated separately for each TNT condition and valence category. Session 1 SCRs were then subtracted from Session 2 SCRs to generate SCR difference scores (dSCR). For details about equipment and data processing, see Methodological Details S1 in the Supplemental Material.

Heart rate variability. Electrocardiography recordings were administered before and after the TNT assessment phase for HRV analysis. We calculated LF-HRV, which reflects both sympathetic and parasympathetic control over heart rate, and HF-HRV, which reflects parasympathetic or vagal modulation (Laborde, Mosley, \& Thayer, 2017). For details about equipment and data processing, see Methodological Details S2 in the Supplemental Material.

Polysomnography. Polysomnography data were acquired to ensure that participants in the sleep group obtained an adequate amount of sleep and to allow us to characterize their sleep architecture (see Methodological Details S3 and Table S1 in the Supplemental Material).

\section{Data analysis}

Intrusion control measures were analyzed for no-think trials with a 2 (Valence: negative/neutral) $\times 2$ (Group: sleep/sleep deprivation) mixed analysis of variance (ANOVA). Analyses of intrusion proportion and relapse probability included the additional factors trial block (1-5) and trial transition (1 to 2,2 to $3, \ldots 9$ to 10 ), respectively. Affect suppression (subjective ratings and SCRs) was analyzed for negative scenes with a 2 (TNT condition: no-think/baseline) $\times 2$ (Group: sleep/sleep deprivation) mixed ANOVA. HRV measures were analyzed using a 2 (Time: before TNT/after TNT) $\times 2$ (Group: sleep/sleep deprivation) ANOVA. Note that ANOVA is robust against deviations from normality (Glass, Peckham, \& Sanders, 1972; Harwell, Rubinstein, Hayes, \& Olds, 1992; Schmider, Ziegler, Danay, Beyer, \& Bühner, 2010). As appropriate, ANOVAs were followed up with post hoc $t$ tests. All reported correlations used Pearson's correlation coefficient. Correlations were compared using Fisher's $z$ transformation.

\section{Results}

\section{Sleep deprivation impairs the suppression of intrusive thoughts}

Overall intrusion scores were robustly higher for think trials $(M=92.76 \%$ of trials, $S E M=1.38 \%)$ compared with no-think trials ( $M=28.87 \%$ of trials, $S E M=2.52 \%$ ), $t(58)=19.73, p<.001, d_{z}=2.57$, confirming that participants largely succeeded in suppressing thoughts about the scenes associated with red-framed faces. Because our primary goal was to examine intrusions for scenes that participants attempted to suppress, our analyses focus on no-think trials hereafter unless otherwise stated. Note that intrusion-proportion scores for no-think trials on the mock TNT task did not differ between groups (sleep group: $M=41.38 \%, S E M=$ $3.76 \%$; sleep-deprivation group: $M=42.22 \%$, SEM $=$ $2.62 \%), t(57)=0.19, p=.854$, suggesting that any between-group differences in intrusion proportion for the TNT assessment phase proper were caused by our sleep/wake manipulation.

Of key interest is whether depriving participants of sleep disrupted their ability to stop unwanted thoughts from coming to mind given reminders. Critically, sleepdeprived participants reported more intrusions than well-rested individuals, $F(1,57)=5.55, p=.022, \eta_{p}{ }^{2}=$ .09 , demonstrating that sleep deprivation impairs memory control (Fig. 2a). Strikingly, the sleep-deprivation 
group suffered a proportional increase in intrusions of nearly $50 \%$ relative to the sleep group, revealing how deficient control may be a pathway to hyperaccessible thoughts. Participants overall showed decreasing intrusions across TNT trial blocks, $F(3.16,180.33)=29.81$, $p<.001, \eta_{p}^{2}=.34$, Greenhouse-Geisser corrected (Fig. $2 \mathrm{~b}$ ), indicating that repeatedly inhibiting retrieval was, in general, highly effective at stopping memories from intruding on future trials. Intrusion rates were comparable for negative and neutral scenes, $F(1,57)=0.11$, $p=.738$, and the detrimental impact of sleep deprivation on intrusion rate did not vary with the valence of scenes $(p>.05)$.

Although suppression robustly reduced intrusions over blocks across all participants, we were also interested in examining whether sleep deprivation altered the rate at which suppression attempts led intrusions to decline. Consistent with deficient inhibitory control, depriving participants of sleep significantly disrupted their ability to down-regulate intrusive thoughts over blocks compared with participants who had slept, as reflected in lower intrusion slope scores, $F(1,55)=9.38$, $p=.003, \eta_{p}^{2}=.15$ (Fig. 2c). Slope scores did not differ between negative and neutral scenes, $F(1,55)=0.08$, $p=.777$, and the intrusion slope deficit in the sleepdeprivation group did not vary with scene valence (p>.05).

We next considered whether sleep-deprived participants were more vulnerable to intrusion relapses for scenes that they had previously suppressed compared with participants that had slept. Sleep-deprived participants exhibited significantly higher relapse probability scores than did the sleep group, $F(1,57)=7.08, p=$ $.010, \eta_{p}^{2}=.11$ (Fig. $2 \mathrm{~d}$ ), indicating that after initially gaining control over an intrusive thought, they suffered intrusion relapses more than participants who had slept. As with intrusions overall, relapses also became less frequent across trial transitions, $F(6.39,364.12)=$ $2.49, p=.020, \eta_{p}^{2}=.04$, Greenhouse-Geisser corrected (Fig. 2e). Relapse probability was comparable for negative and neutral memories, $F(1,57)=0.07, p=.800$, and sleep deprivation affected negative and neutral relapses to a similar degree $(p>.05)$.

\section{Sleep deprivation nullifies affect suppression}

Although the foregoing findings show that sleep deprivation disrupts intrusion control, they do not address whether losing sleep alters how suppression affects emotional responses. Recent work indicates that successfully suppressing negative scenes renders them less aversive when they are later reencountered (Gagnepain et al., 2017). Building on this finding, we tested whether sleep deprivation affected the relationship between retrieval suppression and our behavioral index of affect suppression. Behavioral affect suppression refers to the difference in affect ratings of scenes between the first (before delay and TNT) and second (after delay and TNT) session. Positive affect suppression scores indicate that participants felt less negative about the scenes in the second affect-evaluation task.

Consistent with earlier reports using this procedure (Gagnepain et al., 2017), we found that across all participants, successful retrieval suppression (i.e., fewer intrusions) was associated with greater affect suppression for negative scenes, $r=-.32, p=.015$ (Fig. 3a). In other words, greater success at suppressing retrieval of aversive memories predicted a larger change in their perceived affect, rendering them less negative when participants next encountered the scenes. We observed no such relationship for neutral no-think scenes or think scenes of either valence type (all $p s>.05$ ). The relationship between intrusion control and affect suppression did not differ significantly between the sleep $(r=-.33, p=.079)$ and sleep-deprivation groups $(r=$ $-.17, p=.367 ; z=0.62, p=.533)$.

Having found evidence of a general relationship between intrusion control and affect suppression specifically for negative no-think scenes, we next tested whether sleep deprivation undermines suppressioninduced regulation of negative affect for aversive scenes. We observed a significant interaction between TNT condition (baseline, no-think) and group, $F(1,57)=5.38$, $p=.024, \eta_{p}^{2}=.09$. Affect change scores for aversive no-think scenes were greater in the sleep group than the sleep-deprivation group, $t(57)=2.56, p=.013, d=$ 0.67 (Fig. 3b), supporting the view that the affective benefits of negative thought suppression were impaired after sleep loss. Note that we observed no such difference for negative baseline scenes, $t(57)=0.24, p=.809$, $d=0.06$, suggesting that the effect for negative no-think scenes was not driven by between-group differences in mood. In the sleep group, affect change scores for negative no-think scenes were more positive than those for negative baseline scenes, although this difference did not reach statistical significance, $t(28)=1.76, p=$ $.089, d_{\mathrm{z}}=0.33$. We observed no such difference in the sleep-deprivation group, $t(29)=1.50, p=.145 ; d_{z}=$ 0.27 .

The foregoing findings indicate that suppression reduced the perceived valence of suppressed negative scenes. However, they do not speak to whether suppression alters sympathetic arousal and whether sleep loss affects this form of regulation. To probe this question, we analyzed difference in SCRs (dSCRs) across sessions arising for negative baseline and no-think scenes. Negative dSCR scores reflect a decrease in 
a

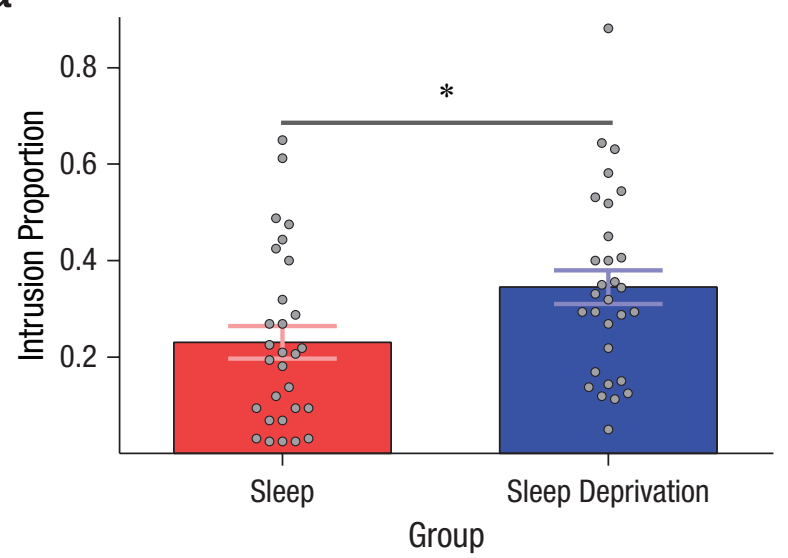

b

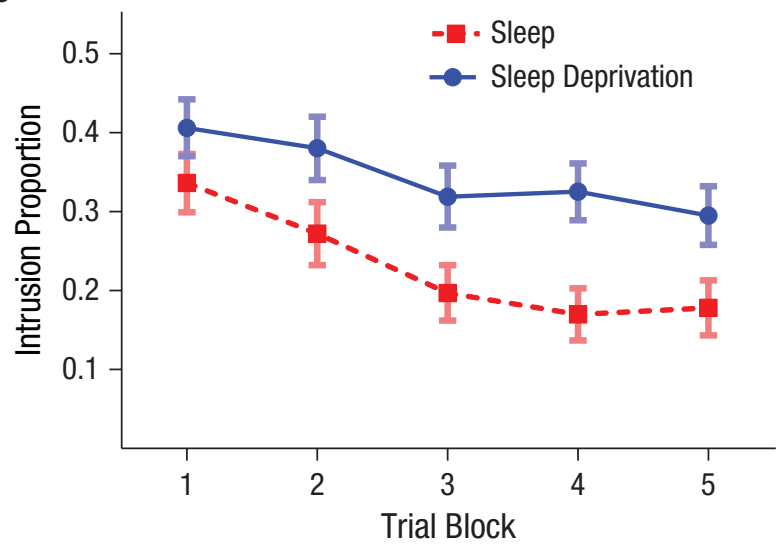

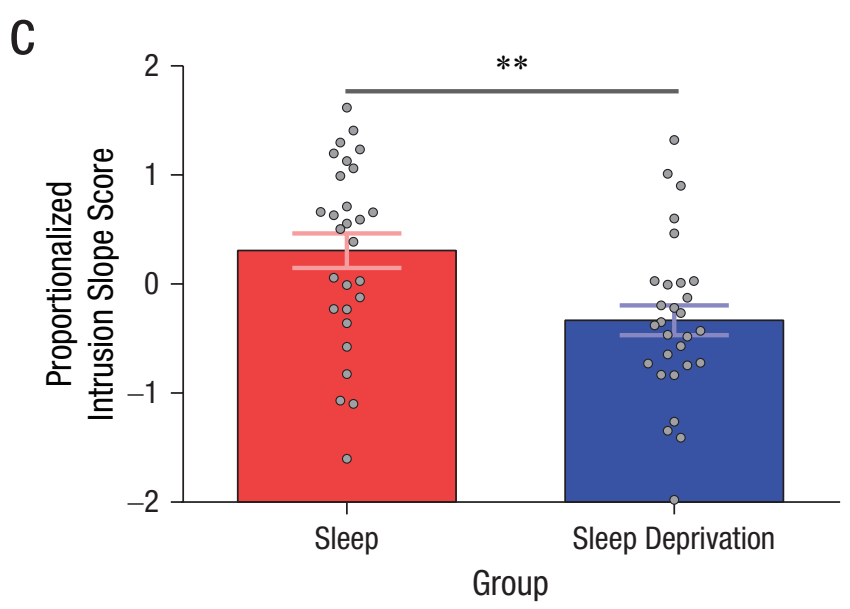

d

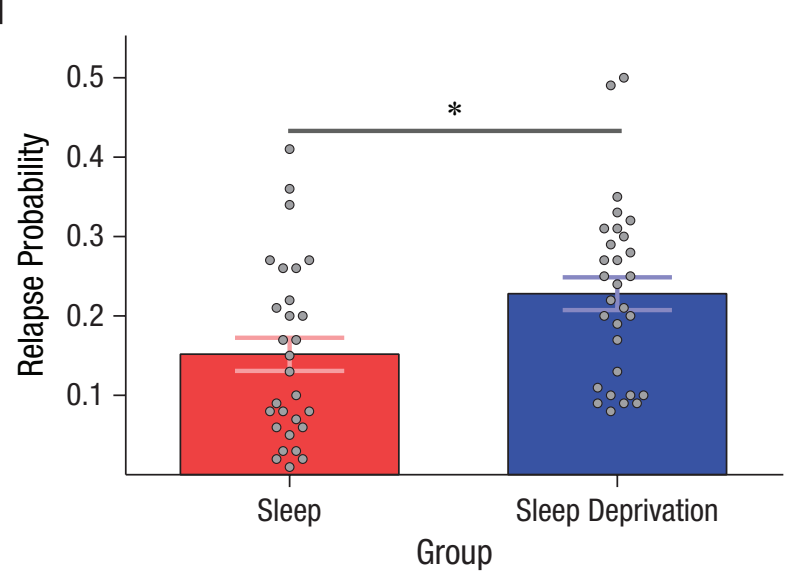

e

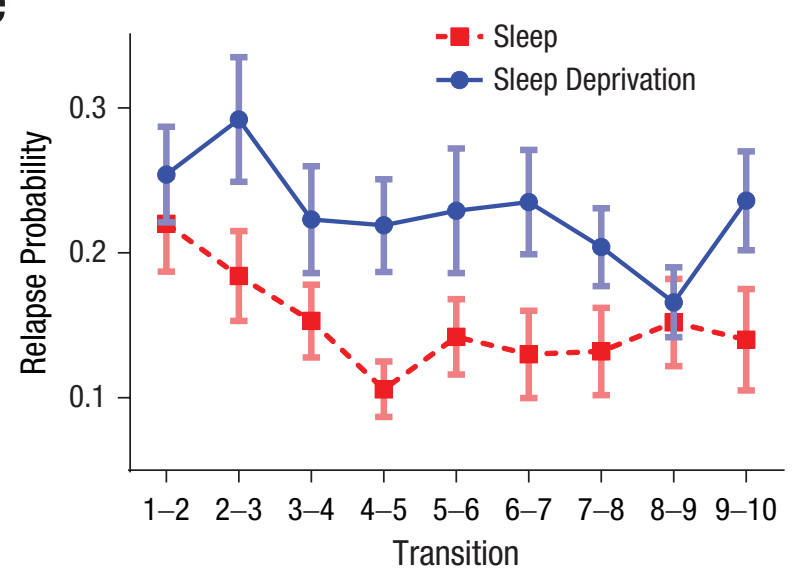

Fig. 2. Sleep deprivation impairs the suppression of intrusive thoughts. Graph in (a) shows intrusion-proportion scores (i.e., the proportion of no-think trials for which participants reported awareness of the associated scene), averaged across think/no-think (TNT) blocks and presented separately for sleep and sleep-deprivation groups. Graph in (b) shows intrusion-proportion scores in each TNT block, presented separately for sleep and sleep-deprivation groups. Graph in (c) shows proportionalized intrusion slope scores (i.e., the rate at which intrusions declined across the five TNT blocks for no-think items, divided by the number of intrusions in the first TNT block), presented separately for sleep and sleep-deprivation groups. Greater slope scores reflect more effective down-regulation of intrusions across trial blocks. Graph in (d) shows relapse-probability scores (i.e., proportion of no-think trials in which successful control on repetition $N$ was followed by failed control on repetition $N+1$ ), averaged across trial transitions and presented separately for sleep and sleep-deprivation groups. Graph in (e) shows relapse-probability scores for each trial transition. Gray dots in (a), (c), and (d) represent individual participants. Asterisks in (a), (c), and (d) indicate significant differences between groups $(* p \leq .05, * * p \leq .01)$. Error bars indicate \pm 1 SEM. 
a

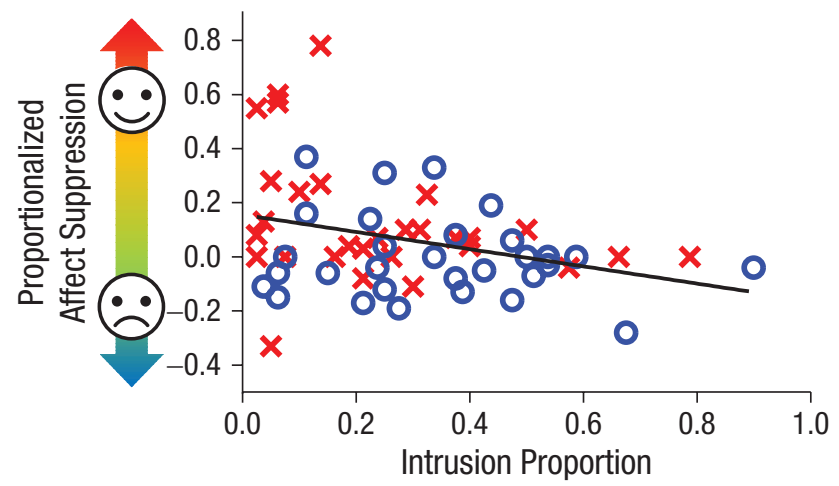

b

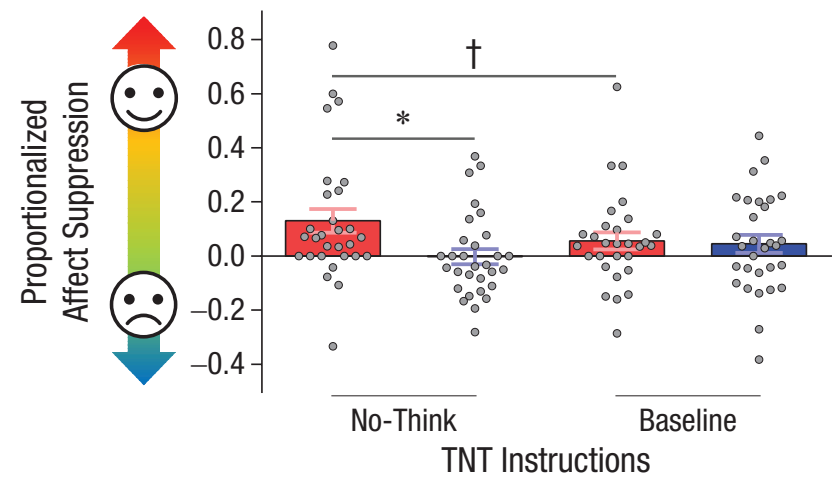

C

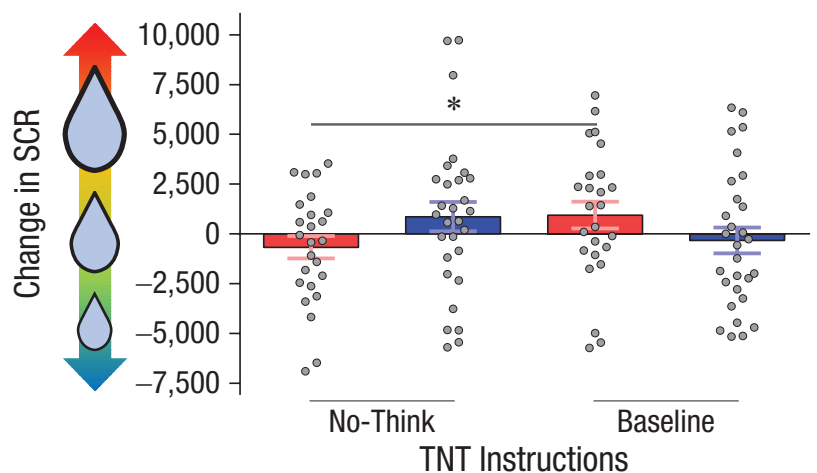

d

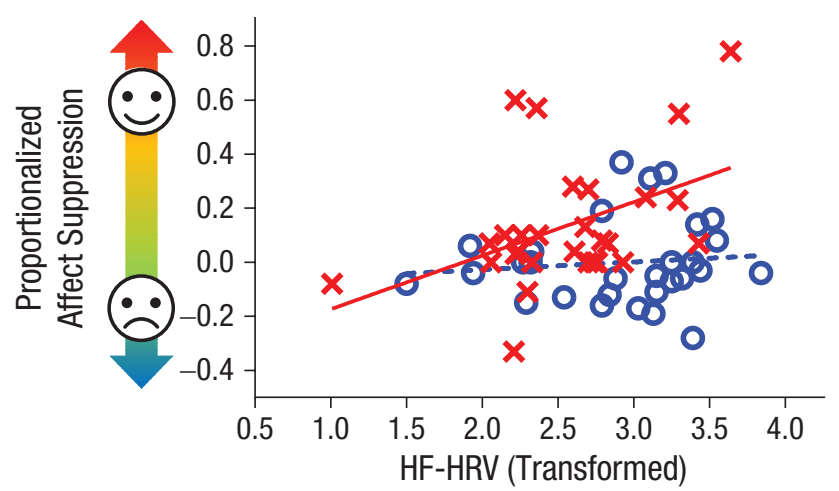

e

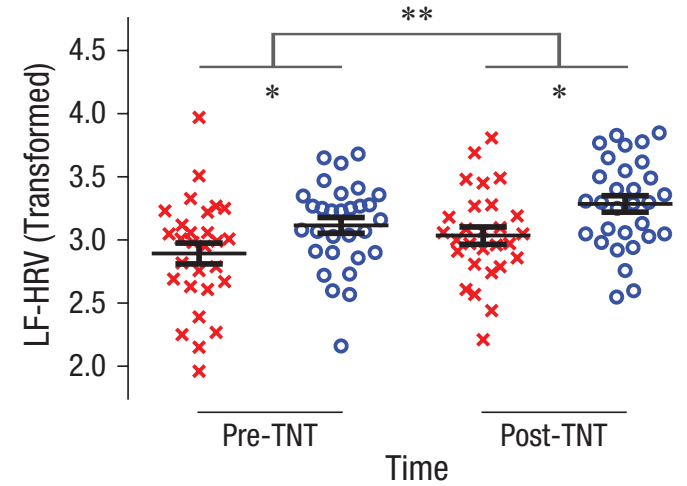

Fig. 3. Sleep deprivation influences affect suppression and heart rate variability. Scatterplot (a; with best-fitting regression line) shows the correlation between intrusion-proportion scores and affect-suppression scores for negative no-think scenes. Graph in (b) shows affect-suppression scores for negative scenes. Graph in (c) shows the change in skin-conductance response (dSCR) toward negative scenes. Scatterplot (d; with best-fitting regression lines) shows the correlation between log-transformed resting high-frequency heart rate variability (HF-HRV; before think/no-think [TNT] task) and affect suppression for negative no-think scenes. Graph in (e) shows mean log-transformed low-frequency (LF) HRV in each group. Data points represent individual participants; error bars in (b), (c), and (e) indicate \pm 1 SEM. Symbols represent main effects of group (b and c; sleep, sleep deprivation) and time (e; before TNT, after TNT; $\left.{ }^{\dagger} p \leq .10, * p \leq .05, * * p \leq .001\right)$.

SCRs toward the scenes in the second affect-evaluation task.

As with our behavioral index of affect suppression, we observed a significant interaction between TNT condition (baseline, no-think) and group on dSCR scores,
$F(1,52)=4.05, p=.049, \eta_{p}{ }^{2}=.07$ (Fig. 3c). Participants who slept exhibited a decrease in dSCR toward the negative no-think scenes compared with baseline scenes, $t(24)=2.07, p=.050, d=0.41$. This finding is consistent with our behavioral affect suppression data 
and further supports the view that successfully suppressing unpleasant memories alleviates their associated emotional charge. Sleep-deprived participants, who were less competent at controlling intrusions, exhibited comparable dSCR scores for baseline and no-think scenes, $t(28)=1.08, p=.290$. These findings suggest that the affective benefits of retrieval suppression for rested participants were not confined to subjective reports of perceived affect but also arose for indices of psychophysiological emotional arousal. Sleep deprivation abolished the affective benefits of suppression, and this was evident in both our behavioral and psychophysiological measures. We observed no reliable correlation between individual differences in our behavioral and psychophysiological affect suppression measures for negative no-think scenes, suggesting that these indices tap partially distinct processes $(r=.09$, $p=.522$ ). The observed change in psychophysiological reactivity for no-think scenes in the sleep group likely reflects diminished sympathetic nervous system responses to suppressed stimuli, possibly initiated by the impact of retrieval suppression on activity in the amygdala (Gagnepain et al., 2017).

\section{Heart rate variability is linked to affect suppression and fatigue}

Inhibitory control over cognition is linked to HF-HRV (Gillie et al., 2014). This finding led us to investigate whether the degree of affect suppression that a given participant could achieve is linked to that participant's HF-HRV and whether any relationship is altered by sleep loss. Indeed, higher HF-HRV (collected before the TNT phase) predicted greater affect suppression scores for negative no-think scenes in the sleep group $(r=$ $.44, p=.021$; Fig. 3d). This relationship was absent in sleep-deprived participants, suggesting that sleep deprivation may reduce the involvement of control processes related to HF-HRV ( $r=.11, p=.577 ; z=1.30, p=.097)$. We observed the same pattern using the HF-HRV measure collected after the TNT phase, although to a lesser degree (sleep group: $r=.34, p=.077$; sleep-deprivation group: $r=.07, p=.724 ; z=1.03, p=.152$ ). In contrast, we found no relationship between HF-HRV (before TNT or after TNT) and affect changes for negative think or baseline scenes or for neutral scenes in any TNT condition for either group (all $p s>.05$ ). We found no relationship between HF-HRV and overall intrusion proportion for no-think scenes (both groups, $p>.05$ ). The LF-HRV component was not associated with affect suppression or overall intrusion proportion in any TNT condition for either group (all $p$ s $>.05$ ).

LF-HRV has been related to both sleep deprivation (Zhong, 2005) and mental fatigue (Egelund, 1982; Tran et al., 2009; Zhao, Zhao, Liu, \& Zheng, 2012). Confirming this relationship, the sleep-deprivation group exhibited higher LF-HRV than the sleep group, $F(1,56)=$ 6.71, $p=.012, \eta_{p}^{2}=.11$ (Fig. 3e). Along similar lines, we found significantly higher LF-HRV following the TNT phase than we did preceding it, $F(1,56)=17.96, p<$ $.001, \eta_{p}^{2}=.24$, reflecting task-induced fatigue, given the $\sim 40$ min duration of the TNT task (van Schie \& Anderson, 2017). We observed no interaction between factors $(p>.05)$.

Performing the TNT task also affected HF-HRV: HFHRV was higher after the TNT task than before it, $F(1$, $56)=19.62, p<.001, \eta_{p}{ }^{2}=.26$. An interaction between time and group, $F(1,56)=9.39, p=.003, \eta_{p}^{2}=.14$, drove this difference. Whereas completing the TNT task elevated HF-HRV in the sleep group, $t(27)=5.54, p<$ $.001, d_{\mathrm{z}}=1.05$, it did not in the sleep-deprivation group, $t(29)=0.93, p=.358, d_{\mathrm{z}}=0.17$, primarily because HFHRV was already high at the outset in sleep-deprived participants. Overall, the groups did not differ reliably in HF-HRV, $F(1,56)=2.26, p=.138$. These data suggest that fatigue may increase HF-HRV but less reliably than LF-HRV.

\section{Discussion}

Our findings indicate that sleep deprivation substantially increases people's vulnerability to unwanted memories intruding into conscious awareness when they confront reminders. Despite exhibiting comparable intrusion control ability before the overnight interval, sleep-deprived participants exhibited a near 50\% proportional increase in intrusions relative to participants that had slept. Moreover, sleep deprivation diminished the cumulative benefits of retrieval suppression for down-regulating subsequent intrusions. Even after sleep-deprived participants initially gained control over unwanted memories and prevented them from intruding, they were consistently more susceptible to relapses when reminders were confronted again later compared with rested individuals.

Deficient memory suppression following sleep loss might arise from dysfunction of the neural networks that govern inhibitory control. Retrieval suppression engages the rDLPFC, which is thought to down-regulate recollection-related activity in MTL areas via inhibitory, top-down mechanisms (Benoit et al., 2015; Gagnepain et al., 2017; Levy \& Anderson, 2012). The functional integrity of the rDLPFC may be particularly vulnerable to sleep loss (Mazur et al., 2002). Functional connectivity from the medial PFC (mPFC) to MTL appears to be disrupted in sleep-deprived individuals when viewing negative emotional images, potentially compromising a pathway of inhibitory control over affect (Yoo et al., 
2007). Moreover, prolonged sleep restriction impairs performance on attention and working memory tasks that rely on prefrontal engagement (Chee \& Choo, 2004; Frenda \& Fenn, 2016; Lim \& Dinges, 2008; Thomas et al., 2000). In the current study, sleep deprivation may have disrupted functional interactions between the rDLPFC (and possibly mPFC) and MTL structures such as the hippocampus and amygdala during retrieval suppression, impairing inhibitory control over memory and affect, increasing intrusions, and decreasing affect suppression. Future research can employ functional brain imaging to address these hypotheses.

Note that sleep deprivation also disrupted people's ability to reduce the affective content of intruding thoughts through retrieval suppression. Consistent with earlier research (Gagnepain et al., 2017), we first confirmed that the more successful people are at controlling unpleasant intrusions, the greater their behavioral affect suppression for the suppressed scenes. Extending this finding, we showed that in rested participants, affect suppression effects arose in psychophysiological reactivity, with suppressed scenes eliciting significantly reduced SCRs compared with scenes that were not suppressed, consistent with a reduction in sympathetic arousal. These behavioral and psychophysiological effects complement prior work showing that indices of affect suppression are predicted by down-regulation of amygdala activity by prefrontal cortex during memory intrusions (Gagnepain et al., 2017), a process that could underlie both effects. Note that sleep-deprived participants showed markedly less behavioral and psychophysiological affect suppression for negative no-think scenes compared with rested participants. These findings suggest that difficulties in engaging inhibitory control to regulate unpleasant intrusions after sleep loss lead to diminished affect regulation for the suppressed content.

The possibility that sleep deprivation compromised affect suppression by altering prefrontal control involvement received indirect support from psychophysiological indices. Among participants who slept normally, higher HF-HRV predicted better affect suppression for negative no-think scenes; this relationship was not found, however, after sleep deprivation. Prior evidence links higher HF-HRV to better executive functioning (for review, see Thayer \& Lane, 2009), including memory control (Gillie et al., 2014). HF-HRV has been hypothesized to reflect the engagement of brain regions supporting cognitive and affective down-regulation during memory inhibition, and in healthy participants, blood flow in the right prefrontal cortex correlates with HF-HRV (Lane et al., 2009). Note, however, in the current data, higher HF-HRV did not predict fewer intrusions for no-think scenes. The selective relationship of HF-HRV and affect suppression may indicate that this measure better indexes the efficiency of those components of the inhibitory control pathway that are uniquely tied to affect regulation (e.g., connectivity between mPFC and amygdala: Yoo et al., 2007). In the sleep-deprivation group, extreme fatigue may have compromised these components, eliminating the relationship between HF-HRV and the emotional aftereffects of suppression, a possibility supported by research on the relationship between sleep deprivation, HF-HRV, and cognitive performance (Quintana et al., 2017).

Prior research suggests that in healthy participants, acute sleep deprivation increases LF-HRV (Zhong, 2005). Replicating this work, our sleep-deprived participants exhibited higher LF-HRV compared with participants who slept. LF-HRV is produced by both sympathetic and parasympathetic systems, whereas HFHRV reflects parasympathetic activity alone (Shaffer \& Ginsberg, 2017). Given that HF-HRV was not altered by our sleep/wake manipulation, our results suggest that sleep deprivation elevates sympathetic arousal. Consistent with earlier work showing that mental fatigue can increase LF-HRV (Egelund, 1982; Tran et al., 2009; Zhao et al., 2012), completing our cognitively demanding TNT task also elevated LF-HRV.

Negative scenes were no more intrusive than neutral scenes despite a highly robust difference in perceived valence reported by our participants (see Method section). This finding might at first seem counterintuitive. However, following previous work that observed the same result (Gagnepain et al., 2017), we matched the initial training level of negative and neutral materials, which may have nullified the benefits of emotional arousal on encoding and consolidation (Canli, Zhao, Brewer, Gabrieli, \& Cahill, 2000; Ritchey, Dolcos, \& Cabeza, 2008). Failure to match initial training of negative and neutral materials may explain prior mixed findings regarding the relation between valence and forgetting. Whereas some studies reported enhanced suppression for negative relative to neutral stimuli (Depue, Banich, \& Curran, 2006; Lambert, Good, \& Kirk, 2010), others observed no difference (van Schie, Geraerts, \& Anderson, 2013) or even the opposite effect (Nørby, Lange, \& Larsen, 2010).

Although impaired memory control following sleep deprivation is a valid interpretation of our data, another possibility relates to overnight memory processing. Previous work has shown that sleep promotes the consolidation of procedural skills (for a review, see Stickgold, 2005). Accordingly, intrusion control may have been impaired in the sleep-deprivation group because these participants did not have an opportunity for 
sleep-associated consolidation of the mnemonic suppression skills learned during the evening TNT practice. Although this interpretation is possible, participants were given only very brief practice with the TNT task (12 no-think trials on six filler items) in the first session, making it an unlikely source of the substantial sleep advantage in intrusion control that we observed. Nevertheless, future work should seek to distinguish the relative contributions of procedural memory consolidation and disrupted inhibitory control to greater intrusiveness after sleep deprivation.

Prior work suggests that sleep curtails spontaneous intrusive thoughts following trauma. Indeed, sleeping soon after exposure to a traumatic film clip reduces the number of spontaneous intrusions relating to that clip in the following week (Kleim, Wysokowsky, Schmid, Seifritz, \& Rasch, 2016). Furthermore, greater sleep disturbance following exposure to a road traffic accident predicts more frequent spontaneous, accident-related thoughts during the first week after trauma (Luik, Iyadurai, Gebhardt, \& Holmes, 2019). Although these studies associated sleep loss with variations in intrusion frequency following adverse events, they did not address the mechanistic basis of these associations. Sleep following a traumatic film, for example, could reduce intrusions over the following week by enabling better memory control or, alternatively, by facilitating sleep-related changes to the memory trace that make it less likely to intrude. Thus, prior work has not established causal evidence concerning whether sleep deprivation impairs retrieval suppression of specific, unwanted thoughts. Because we used a procedure that demands the suppression of aversive memories in response to reminders, the current study pointedly targets the causal status of sleep loss in compromising inhibitory control over thought.

Our findings may help to explain the relationship between sleep disturbance and vulnerability to psychiatric conditions associated with intrusive thoughts. Up to $90 \%$ of patients with PTSD (Maher et al., 2006) and MDD (Riemann et al., 2001) report recurrent sleep disturbances, which are major risk factors for disorder onset (Maher et al., 2006; Mendlewicz, 2009). Exactly how disturbed sleep contributes to these disorders remains elusive, however. Our data raise the possibility that poor intrusion control may help bridge the gap between disturbed sleep and psychiatric symptoms: Insufficient sleep might increase memory intrusions while also nullifying the benefits of retrieval suppression for regulating affect. The onset of intrusive thoughts and affective dysfunction following bouts of poor sleep could create a vicious cycle whereby upsetting intrusions and emotional distress exacerbate sleep problems (Talamini,
Bringmann, de Boer, \& Hofman, 2013), inhibiting the sleep needed to support recovery.

Besides PTSD and MDD, our findings might have implications for understanding other disorders linked to sleep disturbances, such as obsessive compulsive disorder (OCD) and schizophrenia (Coles, Schubert, \& Sharkey, 2012; Monti \& Monti, 2005; Paterson, Reynolds, Ferguson, \& Dawson, 2013). Acute sleep deprivation can induce dissociative symptoms in healthy individuals (Giesbrecht, Smeets, Leppink, Jelicic, \& Merckelbach, 2007; Van Heugten-Van Der Kloet, Giesbrecht, \& Merckelbach, 2015), and sleepiness exacerbates dissociative symptoms in depersonalization disorder (Simeon \& Abugel, 2006). Observations such as these have led researchers to hypothesize that sleep disturbances and associated variables (e.g., intrusive, dream-like mentation during wakefulness) might fuel psychiatric disorders that are linked to dissociation (Lynn et al., 2019; van der Kloet, Merckelbach, Giesbrecht, \& Lynn, 2012). Although dissociative symptoms are commonplace in OCD and schizophrenia, these conditions are also characterized by recurrent and persistent thought intrusions. Our data suggest that sleep loss could contribute to mental illness not only by fostering dissociative symptoms but also by hindering thought suppression mechanisms. It should be noted, however, that a night of total sleep deprivation, as employed in this study, is qualitatively different to the chronic sleep disturbances often observed in psychiatric conditions. Further studies are required to ascertain whether chronic sleep disturbance has similar consequences to acute sleep deprivation for intrusion control and affect suppression.

Evidence suggests that practice improves intrusion control in both real-life situations and via strategic intervention. For example, undergraduate students who report having experienced moderate childhood adversity exhibit greater memory control than those who report experiencing little to no adversity (Hulbert \& Anderson, 2018). This finding suggests that prior experience in suppressing upsetting memories facilitates intrusion control even for new, unrelated memories, leading to greater resilience to adversities later in life. Moreover, providing MDD patients with a cognitive strategy to aid their intrusion control before performing a retrieval suppression task significantly facilitates memory inhibition (Joormann, LeMoult, Hertel, \& Gotlib, 2009). These observations demonstrate that therapeutic strategies targeted at nurturing intrusion control could be used to prevent the development of psychiatric disorders in those who are at risk on account of their sleep problems, such as insomnia sufferers (Baglioni et al., 2011).

Although our study was framed to participants as an investigation of sleep and attention, the use of subjective 
intrusion reports might have given rise to demand characteristics. We must stress, however, that thought intrusions (as measured via subjective intrusion reports) have been consistently linked to objective neural indices (e.g., fMRI: Benoit et al., 2015; Gagnepain et al., 2017; Levy \& Anderson, 2012; EEG: Castiglione et al., 2019; Hellerstedt et al., 2016) as well as later memory performance (Hellerstedt et al., 2016; Levy \& Anderson, 2012). Moreover, consistent with earlier work (Gagnepain et al., 2017), we found that the frequency of reported intrusions correlated with changes in affect evaluation, an effect that is unlikely to have been driven solely by demand characteristics. Future studies should nevertheless incorporate physiological measures to support the validity of intrusion ratings or evaluate demand characteristics by probing participants' perceptions of the study hypotheses in relation to thought intrusions.

In sum, we found that sleep deprivation markedly impairs the ability to prevent unwanted memories from entering conscious thought. Moreover, whereas suppressing negative memories after sleep renders them less subjectively and psychophysiologically aversive, poor intrusion control following sleep deprivation negates this affective benefit. Together with an increasingly specific understanding of the neural machinery of retrieval suppression (Anderson, Bunce, \& Barbas, 2016; Anderson \& Hanslmayr, 2014; Guo, Schmitz, Mur, Ferreira, \& Anderson, 2018; Schmitz, Correia, Ferreira, Prescot, \& Anderson, 2017), these findings point to an important neurocognitive mechanism linking sleep problems to the pathogenesis and maintenance of psychiatric conditions characterized by intrusive symptoms. Developing interventions that improve retrieval suppression in poor sleepers may be a promising avenue for averting the potentially pathogenic consequences of disordered control over distracting thoughts.

\section{Transparency}

Action Editor: Scott O. Lilienfeld

Editor: Scott O. Lilienfeld

Author Contributions

M. O. Harrington, S. A. Cairney, and M. C. Anderson designed the study. M. O. Harrington and J. E. Ashton performed the experiments. M. O. Harrington S. Sankarasubramanian, M. C. Anderson, and S. A. Cairney analyzed the data. M. O. Harrington wrote the manuscript, and all the authors revised the manuscript. All of the authors approved the final manuscript for submission.

Declaration of Conflicting Interests

The author(s) declared that there were no conflicts of interest with respect to the authorship or the publication of this article.

\section{Funding}

This work was supported by Medical Research Council Career Development Award MR-P020208-1 (to S. A. Cairney) and Medical Research Council Grant MC-A060-5PR00 (to M. C. Anderson).

Open Practices

All data have been made publicly available via OSF and can be accessed at https://osf.io/apv8y/. The complete Open Practices Disclosure for this article can be found at http://journals.sagepub.com/doi/suppl/10.1177/ 2167702620951511. This article has received the badge for Open Data. More information about the Open Practices badges can be found at https://www.psychologicalscience .org/publications/badges.

(I)

\section{ORCID iD}

Marcus O. Harrington (iD https://orcid.org/0000-0002-6292-7595

\section{Acknowledgments}

We are grateful to Rhiannon Pearce, Tomas Vaitkus, and Hanna Weiers for their assistance with data collection and to Steven J. Lynn and another anonymous reviewer for their helpful comments on an earlier version of the manuscript.

\section{Supplemental Material}

Additional supporting information can be found at http:// journals.sagepub.com/doi/suppl/10.1177/2167702620951511

\section{References}

Alhola, P., \& Polo-Kantola, P. (2007). Sleep deprivation: Impact on cognitive performance. Neuropsychiatric Disease and Treatment, 3, 553-567. doi:10.1016/j.smrv.2012.06 .007

Anderson, M. C., Bunce, J. G., \& Barbas, H. (2016). Prefrontalhippocampal pathways underlying inhibitory control over memory. Neurobiology of Learning and Memory, 134, 145-161. doi:10.1016/j.nlm.2015.11.008

Anderson, M. C., \& Green, C. (2001). Suppressing unwanted memories by executive control. Nature, 410(6826), 366369. doi: $10.1038 / 35066572$

Anderson, M. C., \& Hanslmayr, S. (2014). Neural mechanisms of motivated forgetting. Trends in Cognitive Sciences, 18, 279-282. doi:10.1016/j.tics.2014.03.002

Baglioni, C., Battagliese, G., Feige, B., Spiegelhalder, K., Nissen, C., Voderholzer, U., ... Riemann, D. (2011). Insomnia as a predictor of depression: A meta-analytic evaluation of longitudinal epidemiological studies. Journal of Affective Disorders, 135, 10-19. doi:10.1016/j .jad.2011.01.011

Beck, A. T., Steer, R. A., \& Brown, G. K. (1996). Manual for the Beck depression inventory-II. San Antonio, TX: Psychological Corporation.

Benoit, R. G., Hulbert, J. C., Huddleston, E., \& Anderson, M. C. (2015). Adaptive top-down suppression of hippocampal activity and the purging of intrusive memories from consciousness. Journal of Cognitive Neuroscience, 27, 96-111. doi:10.1162/jocn 
Brewin, C. R., Gregory, J. D., Lipton, M., \& Burgess, N. (2010). Intrusive images in psychological disorders: Characteristics, neural mechanisms, and treatment implications. Psychological Review, 117, 210-232. doi:10.1037/ a0018113

Canli, T., Zhao, Z., Brewer, J., Gabrieli, J. D., \& Cahill, L. (2000). Event-related activation in the human amygdala associates with later memory for individual emotional experience. The Journal of Neuroscience, 20(19), RC99. doi: 20004570

Castiglione, A., Wagner, J., Anderson, M., \& Aron, A. R. (2019). Preventing a thought from coming to mind elicits increased right frontal beta just as stopping action does. Cerebral Cortex, 29, 2160-2172. doi:10.1093/cercor/bhz017

Chee, M. W. L. (2004). Functional imaging of working memory after $24 \mathrm{hr}$ of total sleep deprivation. The Journal of Neuroscience, 24, 4560-4567. doi:10.1523/JNEURO SCI.0007-04.2004

Chee, M. W. L., \& Choo, W. C. (2004). Functional imaging of working memory after $24 \mathrm{hr}$ of total sleep deprivation. The Journal of Neuroscience, 24, 4560-4567. doi:10.1523/ JNEUROSCI.0007-04.2004

Coles, M. E., Schubert, J. R., \& Sharkey, K. M. (2012). Delayed bedtimes and obsessive-compulsive symptoms. Behavioral Sleep Medicine, 10, 258-265. doi:10.1080/15 402002.2011 .634939

Depue, B. E., Banich, M. T., \& Curran, T. (2006). Suppression of emotional and nonemotional content in memory: Effects of repetition on cognitive control. Psychological Science, 17, 441-447. doi:10.1111/j.1467-9280.2006.01725.x.Suppression

Dinges, J. D. F., Pack, F., Williams, K., Gillen, K. A., Powell, J. W., Ott, G. E., . . Pack, J. A. I. (1997). Cumulative sleepiness, mood disturbance, and psychomotor vigilance performance decrements during a week of sleep restricted to 4-5 hours per night. Sleep, 20, 267-277. doi:10.1093/ sleep/20.4.267

Drummond, S. P. A., Paulus, M. P., \& Tapert, S. F. (2006). Effects of two nights sleep deprivation and two nights recovery sleep on response inhibition. Journal of Sleep Research, 15, 261-265. doi:10.1111/j.1365-2869.2006.00535.x

Egelund, N. (1982). Spectral analysis of heart rate variability as an indicator of driver fatigue. Ergonomics, 25, 663-672. doi:10.1080/00140138208925026

Frenda, S. J., \& Fenn, K. M. (2016). Sleep less, think worse: The effect of sleep deprivation on working memory. Journal of Applied Research in Memory and Cognition, 5, 463-469. doi:10.1016/j.jarmac.2016.10.001

Gagnepain, P., Hulbert, J., \& Anderson, M. C. (2017). Parallel regulation of memory and emotion supports the suppression of intrusive memories. The Journal of Neuroscience, 37, 6423-6441. doi:10.1523/JNEUROSCI.2732-16.2017

Giesbrecht, T., Smeets, T., Leppink, J., Jelicic, M., \& Merckelbach, H. (2007). Acute dissociation after 1 night of sleep loss. Journal of Abnormal Psychology, 116, 599-606. doi:10.1037/0021-843X.116.3.599

Gillie, B. L., Vasey, M. W., \& Thayer, J. F. (2014). Heart rate variability predicts control over memory retrieval. Psychological Science, 25, 458-465. doi:10.1177/0956797613508789
Glass, G. V, Peckham, P. D., \& Sanders, J. R. (1972). Consequences of failure to meet assumptions underlying the fixed effects analyses of variance and covariance. Review of Educational Research, 42, 237-288. doi:10.3102/003465430 42003237

Guo, Y., Schmitz, T. W., Mur, M., Ferreira, C. S., \& Anderson, M. C. (2018). A supramodal role of the basal ganglia in memory and motor inhibition: Meta-analytic evidence. Neuropsychologia, 108, 117-134. doi:10.1016/j.neuropsy chologia.2017.11.033

Harwell, M. R., Rubinstein, E. N., Hayes, W. S., \& Olds, C. C. (1992). Summarizing Monte Carlo results in methodological research: The one- and two-factor fixed effects ANOVA cases. Journal of Educational and Behavioral Statistics, 17, 315-339. doi:10.3102/10769986017004315

Hellerstedt, R., Johansson, M., \& Anderson, M. C. (2016). Tracking the intrusion of unwanted memories into awareness with event-related potentials. Neuropsychologia, 89, 510-523. doi:10.1016/j.neuropsychologia.2016.07.008

Hulbert, J. C., \& Anderson, M. C. (2018). What doesn't kill you makes you stronger: Psychological trauma and its relationship to enhanced memory control. Journal of Experimental Psychology: General, 147, 1931-1949. doi:10.1037/ xge0000461

Joormann, J., LeMoult, J., Hertel, P. T., \& Gotlib, I. H. (2009). Training forgetting of negative material in depression. Journal of Abnormal Psychology, 118, 34-43. doi:10.1037/ a0013794.Training

Kleim, B., Wysokowsky, J., Schmid, N., Seifritz, E., \& Rasch, B. (2016). Effects of sleep after experimental trauma on intrusive emotional memories. Sleep, 39, 2125-2132. doi: 10.5665/sleep. 6310

Laborde, S., Mosley, E., \& Thayer, J. F. (2017). Heart rate variability and cardiac vagal tone in psychophysiological research - Recommendations for experiment planning, data analysis, and data reporting. Frontiers in Psychology, 8, Article 213. doi:10.3389/fpsyg.2017.00213

Lambert, A. J., Good, K. S., \& Kirk, I. J. (2010). Testing the repression hypothesis: Effects of emotional valence on memory suppression in the think - no think task. Consciousness and Cognition, 19, 281-293. doi:10.1016/j .concog.2009.09.004

Lane, R. D., McRae, K., Reiman, E. M., Chen, K., Ahern, G. L., \& Thayer, J. F. (2009). Neural correlates of heart rate variability during emotion. NeuroImage, 44, 213-222. doi:10.1016/j.neuroimage.2008.07.056

Lang, P. J., Bradley, M. M., \& Cuthbert, B. N. (2005). International Affective Picture System (IAPS): Technical manual and affective ratings. Technical report. Gainesville: University of Florida. doi:10.1027/0269-8803/a000147

Levy, B. J., \& Anderson, M. C. (2008). Individual differences in the suppression of unwanted memories: The executive deficit hypothesis. Acta Psychologica, 127, 623-635. doi:10.1016/j.actpsy.2007.12.004

Levy, B. J., \& Anderson, M. C. (2012). Purging of memories from conscious awareness tracked in the human brain. The Journal of Neuroscience, 32, 16785-16794. doi:10.1523/JNEUROSCI.2640-12.2012 
Lim, J., \& Dinges, D. F. (2008). Sleep deprivation and vigilant attention. Annals of the New York Academy of Sciences, 322, 305-322. doi:10.1196/annals.1417.002

Luik, A. I., Iyadurai, L., Gebhardt, I., \& Holmes, E. A. (2019). Sleep disturbance and intrusive memories after presenting to the emergency department following a traumatic motor vehicle accident: An exploratory analysis. European Journal of Psychotraumatology, 10, 1556550. doi:10.1080/ 20008198.2018.1556550

Lynn, S. J., Maxwell, R., Merckelbach, H., Lilienfeld, S. O., Kloet, D. van H. van der, \& Miskovic, V. (2019). Dissociation and its disorders: Competing models, future directions, and a way forward. Clinical Psychology Review, 73, Article 101755. doi:10.1016/j.cpr.2019.101755

Maher, M. J., Rego, S. A., \& Asnis, G. M. (2006). Sleep disturbances in patients with post-traumatic stress disorder: Epidemiology, impact and approaches to management. CNS Drugs, 20, 567-590. doi:10.2165/00023210200620070-00003

Mary, A., Dayan, J., Leone, G., Postel, C., Fraisse, F., Malle, C., . . . Gagnepain, P. (2020). Resilience after trauma: The role of memory suppression. Science, 367(6479), Artucke eaay8477. doi:10.1126/science.aay8477

Mazur, A., Pace-Schott, E. F., \& Hobson, J. A. (2002). The prefrontal cortex in sleep. Trends in Cognitive Sciences, 6, 475-481. doi:10.1016/S1364-6613(02)01992-7

Mendlewicz, J. (2009). Sleep disturbances: Core symptoms of major depressive disorder rather than associated or comorbid disorders. The World Journal of Biological Psychiatry, 10, 269-275. doi:10.3109/15622970802503086

Mihailova, S., \& Jobson, L. (2018). Association between intrusive negative autobiographical memories and depression: A meta-analytic investigation. Clinical Psychology and Psychotherapy, 25, 509-524. doi:10.1002/cpp.2184

Monti, J. M., \& Monti, D. (2005). Sleep disturbance in schizophrenia. International Review of Psychiatry, 17, 247-253. doi:10.1080/09540260500104516

Moritz, S., Hörmann, C. C., Schröder, J., Berger, T., Jacob, G. A., Meyer, B., . . Klein, J. P. (2014). Beyond words: Sensory properties of depressive thoughts. Cognition and Emotion, 28, 1047-1056. doi:10.1080/02699931.2013.86 8342

Newby, J. M., \& Moulds, M. L. (2011). Characteristics of intrusive memories in a community sample of depressed, recovered depressed and never-depressed individuals. Behaviour Research and Therapy, 49, 234-243. doi:10.1016/j.brat.2011.01.003

Nilsson, J. P., Soderstrom, M., Karlsson, A. U., Lekander, M., Akerstedt, T., Lindroth, N. E., \& Axelsson, J. (2005). Less effective executive functioning after one night's sleep deprivation. Journal of Sleep Research, 14, 1-6. doi:10.1111/j.1365-2869.2005.00442.x

Nørby, S., Lange, M., \& Larsen, A. (2010). Forgetting to forget: On the duration of voluntary suppression of neutral and emotional memories. Acta Psychologica, 133, 73-80. doi:10.1016/j.actpsy.2009.10.002

Paterson, J. L., Reynolds, A. C., Ferguson, S. A., \& Dawson, D. (2013). Sleep and obsessive-compulsive disorder (OCD).
Sleep Medicine Reviews, 17, 465-474. doi:10.1016/j.smrv .2012 .12 .002

Payne, A., Kralj, A., Young, J., \& Meiser-Stedman, R. (2019). The prevalence of intrusive memories in adult depression: A meta-analysis. Journal of Affective Disorders, 153, 193-202. doi:10.1016/j.jad.2019.04.055

Quintana, D. S., Elvsåshagen, T., Zak, N., Norbom, L. B., Pedersen, P., Quraishi, S. H., . . . Westlye, L. T. (2017). Diurnal variation and twenty-four hour sleep deprivation do not alter supine heart rate variability in healthy male young adults. PLOS ONE, 12(2), Article e0170921. doi:10.1371/journal.pone.0170921

Riemann, D., Berger, M., \& Voderholzer, U. (2001). Sleep and depression - Results from psychobiological studies: An overview. Biological Psychology, 57, 67-103. doi:10.1016/ S0301-0511(01)00090-4

Ritchey, M., Dolcos, F., \& Cabeza, R. (2008). Role of amygdala connectivity in the persistence of emotional memories over time: An event-related fMRI investigation. Cerebral Cortex, 18, 2494-2504. doi:10.1093/cercor/bhm262

Schmider, E., Ziegler, M., Danay, E., Beyer, L., \& Bühner, M. (2010). Is it really robust? Reinvestigating the robustness of ANOVA against violations of the normal distribution assumption. Methodology, 6, 147-151. doi:10.1027/16142241/a000016

Schmitz, T. W., Correia, M. M., Ferreira, C. S., Prescot, A. P., \& Anderson, M. C. (2017). Hippocampal GABA enables inhibitory control over unwanted thoughts. Nature Communications, 8, Article 1311. doi:10.1038/s41467-017-00956-z

Shaffer, F., \& Ginsberg, J. P. (2017). An overview of heart rate variability metrics and norms. Frontiers in Public Health, 5, Article 258. doi:10.3389/fpubh.2017.00258

Short, M. A., \& Louca, M. (2015). Sleep deprivation leads to mood deficits in healthy adolescents. Sleep Medicine, 16, 987-993. doi:10.1016/j.sleep.2015.03.007

Simeon, D., \& Abugel, J. (2006). Feeling unreal: Depersonalization disorder and the loss of the self. New York, NY: Oxford University Press.

Stickgold, R. (2005). Sleep-dependent memory consolidation. Nature, 437(7063), 1272-1278. doi:10.1038/nature04286

Streb, M., Mecklinger, A., Anderson, M. C., Johanna, L. H., \& Michael, T. (2016). Memory control ability modulates intrusive memories after analogue trauma. Journal of Affective Disorders, 192, 134-142. doi:10.1016/j.jad .2015 .12 .032

Talamini, L. M., Bringmann, L. F., de Boer, M., \& Hofman, W. F. (2013). Sleeping worries away or worrying away sleep? Physiological evidence on sleep-emotion interactions. PLOS ONE, 8(5), Article e62480. doi:10.1371/jour nal.pone.0062480

Thayer, J. F., \& Lane, R. D. (2009). Claude Bernard and the heart-brain connection: Further elaboration of a model of neurovisceral integration. Neuroscience and Biobehavioral Reviews, 33, 81-88. doi:10.1016/j.neubiorev.2008.08.004

Thomas, M. L., Sing, H. C., Belenky, G., Holcomb, H. H., Mayberg, H. S., Dannals, R. F., ... Redmond, D. P. (2000). Neural basis of alertness and cognitive performance impairments during sleepiness I. Effects of $24 \mathrm{~h}$ of 
sleep deprivation on waking human regional brain activity. Journal of Sleep Research, 9, 335-352. doi:10.1016/ S1472-9288(03)00020-7

Thomas, M. L., Sing, H. C., Belenky, G., Holcomb, H. H., Mayberg, H. S., Dannals, R. F., ... Redmond, D. P. (2003). Neural basis of alertness and cognitive performance impairments during sleepiness II. Effects of 48 and $72 \mathrm{~h}$ of sleep deprivation on waking human regional brain activity. Thalamus and Related Systems, 2, 199-229. doi:10.1016/S1472-9288(03)00020-7

Tran, Y., Wijesuriya, N., Tarvainen, M., Karjalainen, P., \& Craig, A. (2009). The relationship between spectral changes in heart rate variability and fatigue. Journal of Psychophysiology, 23, 143-151. doi:10.1027/0269-8803.23.3.143

van der Kloet, D., Merckelbach, H., Giesbrecht, T., \& Lynn, S. J. (2012). Fragmented sleep, fragmented mind: The Role of Sleep in Dissociative symptoms. Perspectives on Psychological Science, 7, 159-175. doi:10.1177/17456 91612437597

Van Heugten-Van Der Kloet, D., Giesbrecht, T., \& Merckelbach, H. (2015). Sleep loss increases dissociation and affects memory for emotional stimuli. Journal of Behavior Therapy and Experimental Psychiatry, 47, 9-17. doi:10.1016/j.jbtep.2014.11.002

van Schie, K., \& Anderson, M. C. (2017). Successfully controlling intrusive memories is harder when control must be sustained. Memory, 25, 1201-1216. doi:10.1080/0965821 1.2017 .1282518 van Schie, K., Geraerts, E., \& Anderson, M. C. (2013). Emotional and non-emotional memories are suppressible under direct suppression instructions. Cognition and Emotion, 27, 1122-1131. doi:10.1080/02699931.2013.76 5387

Walker, M. P. (2009). The role of sleep in cognition and emotion. Annals of the New York Academy of Sciences, 1156, 168-197. doi:10.1111/j.1749-6632.2009.04416.x

Wild, C. J., Nichols, E. S., Battista, M. E., Stojanoski, B., \& Owen, A. M. (2018). Dissociable effect of self-reported daily sleep duration on high-level cognitive abilities. Sleep, 41(12), Article zsy182. doi:10.1093/sleep/zsy182

Yoo, S. S., Gujar, N., Hu, P., Jolesz, F. A., \& Walker, M. P. (2007). The human emotional brain without sleepa prefrontal amygdala disconnect. Current Biology, 17, 877-878. doi:10.1016/j.cub.2007.08.007

Zhao, C., Zhao, M., Liu, J., \& Zheng, C. (2012). Electroencephalogram and electrocardiograph assessment of mental fatigue in a driving simulator. Accident Analysis and Prevention, 45, 83-90. doi:10.1016/j.aap.2011.11.019

Zhong, X. (2005). Increased sympathetic and decreased parasympathetic cardiovascular modulation in normal humans with acute sleep deprivation. Journal of Applied Physiology, 98, 2024-2032. doi:10.1152/japplphysiol.00620.2004

Zohar, D., Tzischinsky, O., Epstein, R., \& Lavie, P. (2005). The effects of sleep loss on medical residents' emotional reactions to work events: A cognitive-energy model. Sleep, 28, 47-54. doi:10.1093/sleep/28.1.47 